\title{
Cthrc1 Is a Positive Regulator of Osteoblastic Bone Formation
}

\author{
Hiroaki Kimura', Kin Ming Kwan ${ }^{2,4}$, Zhaoping Zhang ${ }^{2}$, Jian Min Deng ${ }^{2}$, Bryant G. Darnay ${ }^{3}$, Richard R. \\ Behringer $^{2}$, Takashi Nakamura', Benoit de Crombrugghe ${ }^{2}$, Haruhiko Akiyama ${ }^{1 *}$
}

1 Department of Orthopaedics, Kyoto University, Kyoto, Japan, 2 Department of Molecular Genetics, The University of Texas M. D. Anderson Cancer Center, Houston, Texas, United States of America, 3 Department of Experimental Therapeutics, The University of Texas M. D. Anderson Cancer Center, Houston, Texas, United States of America, 4 Department of Biology, The Chinese University of Hong Kong, Shatin, Hong Kong SAR, China

\begin{abstract}
Background: Bone mass is maintained by continuous remodeling through repeated cycles of bone resorption by osteoclasts and bone formation by osteoblasts. This remodeling process is regulated by many systemic and local factors.

Methodology/Principal Findings: We identified collagen triple helix repeat containing-1 (Cthrc1) as a downstream target of bone morphogenetic protein-2 (BMP2) in osteochondroprogenitor-like cells by PCR-based suppression subtractive hybridization followed by differential hybridization, and found that Cthrc1 was expressed in bone tissues in vivo. To investigate the role of Cthrc1 in bone, we generated Cthrc1-null mice and transgenic mice which overexpress Cthrc1 in osteoblasts (Cthrc1 transgenic mice). Microcomputed tomography (micro-CT) and bone histomorphometry analyses showed that Cthrc1-null mice displayed low bone mass as a result of decreased osteoblastic bone formation, whereas Cthrc1 transgenic mice displayed high bone mass by increase in osteoblastic bone formation. Osteoblast number was decreased in Cthrc1-null mice, and increased in Cthrc1 transgenic mice, respectively, while osteoclast number had no change in both mutant mice. In vitro, colony-forming unit (CFU) assays in bone marrow cells harvested from Cthrc1-null mice or Cthrc1 transgenic mice revealed that Cthrc1 stimulated differentiation and mineralization of osteoprogenitor cells. Expression levels of osteoblast specific genes, ALP, Col1a1, and Osteocalcin, in primary osteoblasts were decreased in Cthrc1-null mice and increased in Cthrc1 transgenic mice, respectively. Furthermore, BrdU incorporation assays showed that Cthrc1 accelerated osteoblast proliferation in vitro and in vivo. In addition, overexpression of Cthrc1 in the transgenic mice attenuated ovariectomy-induced bone loss.
\end{abstract}

Conclusions/Significance: Our results indicate that Cthrc1 increases bone mass as a positive regulator of osteoblastic bone formation and offers an anabolic approach for the treatment of osteoporosis.

Citation: Kimura H, Kwan KM, Zhang Z, Deng JM, Darnay BG, et al. (2008) Cthrc1 Is a Positive Regulator of Osteoblastic Bone Formation. PLoS ONE 3(9): e3174. doi:10.1371/journal.pone.0003174

Editor: Suzannah Rutherford, Fred Hutchinson Cancer Research Center, United States of America

Received June 28, 2008; Accepted August 16, 2008; Published September 9, 2008

Copyright: ( 2008 Kimura et al. This is an open-access article distributed under the terms of the Creative Commons Attribution License, which permits unrestricted use, distribution, and reproduction in any medium, provided the original author and source are credited.

Funding: This work was supported by a Grant-in-aid for Scientific Research from the Japanese Ministry of Education, Culture, Sports, Science, and Technology (\#18390413).

Competing Interests: The authors have declared that no competing interests exist.

* E-mail: hakiyama@kuhp.kyoto-u.ac.jp

\section{Introduction}

Bone modeling is initiated by osteoblastic differentiation of mesenchymal cells into preosteoblasts. Preosteoblasts then differentiate into functional osteoblasts producing bone matrix proteins, including type I collagen, osteopontin, and osteocalcin to form bone tissues. Bone is constantly remodeled through bone formation by functional osteoblasts and bone resorption by osteoclasts which arise from monocytic cells, and bone mass is maintained by their precise balance [1-3]. Recently, significant advances have been made in our understandings of the factors that regulate the balance between osteoblastic bone formation and osteoclastic bone resorption, but the mechanisms have not been fully elucidated.

Bone morphogenetic proteins (BMPs) are potent osteogenic agents that induce differentiation of mesenchymal cells toward an osteoblastic lineage and stimulate the differentiation and functions of osteoblasts during a process of bone modeling [4-6].
Postnatally, BMP signaling regulates osteoblastic bone formation and osteoclastic bone resorption in bone remodeling [7]. Recent studies show that osteoblast-specific inhibition of BMP signals in mice affects postnatal bone mass [8-12]. Osteoblast-specific Bmprla deficient mice show reduced bone volume and bone formation rates with normal osteoblast number due to impaired osteoblast function at 3 months old and increased bone volume probably due to reduced osteoclastic bone resorption at 10 months old [11]. Transgenic mice that osteoblast-specifically overexpress noggin, the BMP inhibitor, display reduced bone mineral densities and bone formation rates due to impaired osteoblast function $[8,9]$. Mice carrying a targeted deletion of Tob, which is a member of a new antiproliferative protein family and represses BMPinduced Smad-dependent transcription in osteoblasts, have increased bone mass due to increased osteoblast numbers and acceleration of the bone formation rates [12]. Thus, BMPs are key molecules that control bone modeling and bone remodeling. 
To identify the molecules that regulate bone modeling and/or bone remodeling as a downstream target of BMP signaling, we compared the mRNAs expressed in BMP2-untreated and BMP2treated ATDC5 osteochondroprogenitor-like cells by PCR-based suppression subtractive hybridization followed by differential hybridization, and identified a cDNA upregulated by BMP2, that encoded Collagen triple helix repeat containing protein 1 (Cthrc1). Cthrcl is a $30-\mathrm{kD}$ glycosylated secreted protein containing a short collagen-like motif with $12 \mathrm{Gly}-\mathrm{X}-\mathrm{Y}$ repeats similar to the collagen domains present in the $\mathrm{Clq} /$ tumor necrosis factor $\alpha$ related proteins [13]. Cthrc1 is transiently expressed in the arterial wall in response to injury, suggesting that Cthrcl is involved in vascular remodeling by limiting collagen matrix deposition and promoting cell migration [13]. A recent study shows that neointimal lesion formation and adventitial collagen deposition in response to carotid artery ligation are reduced in transgenic mice overexpressing Cthrc1 under the control of cytomegalovirus promoter [14]. According to expression analysis of Cthrc1, abundant expression of Cthrc1 is observed in developing skeleton during embryogenesis and in the bone matrix and periosteum in adult mice [15].

In this study, we generated Cthrc1-null mice and transgenic mice that overexpress Cthrc1 under the control of Colla1 osteoblastspecific promoter. Cthrc1-null mice developed a low-bone-massphenotype as a result of reduced bone formation, while the transgenic mice exhibited a high-bone-mass-phenotype caused by enhanced bone formation. In vitro analyses showed that Cthrcl stimulated osteoblast proliferation and differentiation. Thus, our results indicate that Cthrcl is a positive regulator of osteoblastic bone formation.

\section{Results}

\section{Cthrc1 is expressed in bone}

To identify potential osteoblast-specific proteins as downstreams of BMPs, we performed two-step screening consisting of PCRbased suppression subtractive hybridization followed by differential hybridization using the poly(A) ${ }^{+}$RNA extracted from BMP2untreated and BMP2-treated ATDC5 cells, and identified a cDNA encoding Cthrc1(Figure S1A). In vitro, Cthrc1 was expressed in MC3T3-E1 osteoblastic cells and differentiated ATDC5 cells, but not in C3H10T1/2 fibroblastic cells and C2C12 myoblastic cells (Figure S1B). We next assessed the expression of Cthrc1 in various adult mouse tissues by northern blot analysis, and Cthrc1 was expressed in bone but not in the other adult mouse tissues examined (Figure S1C). During the skeletogenesis in the limb buds of mouse embryos, Cthrc1 was expressed in the mesenchymal condensation in E13.5 and in the bone tissues in E16.5 (Figure S1D). In addition, the expression levels of Cthrc1 increased during mouse embryogenesis (Figure S1E). These results suggest that Cthrcl plays an important role in bone modeling or remodeling.

\section{Low bone mass and decreased bone formation in Cthrc1- null mice}

To investigate the physiological role of Cthrcl in bone, we inactivated the Cthrc1 gene in mouse embryonic stem cells by homologous recombination. In the target strategy, an IRES-lacz$p A$-loxP-flanked neomycin resistance expression cassette was introduced into exon 2 which codes a short Gly-X-Y collagen triple helix repeat domain (Figure $1 \mathrm{~A}$ and $\mathrm{B}$ ). As shown in Figure 1C, Cthrc1 RNA was absent in E16.5 Cthrc1-null embryo, indicating that the mutation was a null mutation. Homozygous Cthrc1 mutant mice were born at the expected Mendelian ratio and grew normally in comparison with their wild-type littermates (data not shown). X-gal staining of heterozygous Cthrc1 mutant embryos revealed the expression of Cthrcl in bone-forming tissues in E12.5 embryos and in bones and periarticular cartilages in E16.5 embryos, which corresponded to the results of in situ hybridization (Figure 1D and Figure S2A). Skeletal preparations showed no obvious skeletal phenotypes in Cthrc1-null newborn mice (Figure S2B). In situ hybridization analyses showed comparable expression of osteoblast marker genes, Runx2 and Col1a1, and chondrocyte marker genes, Col2a1 and Col10a1 between E16.5 Cthrc1-null embryos and their wild-type littermates (Figure S3A), suggesting that Cthrcl has no apparent effect on skeletal development. However, histological analyses of decalcified adult bone tissues revealed that the number and thickness of trabecular bones were reduced in Cthrc1-null mice, compared with their wild-type littermates (data not shown). This result suggested that Cthrcl may regulate bone remodeling postnatally. To test this hypothesis, we performed bone histomorphometric analyses and microcomputed tomography (micro-CT) analyses of 2-month-old Cthrc1-null and wild-type mice. Micro-CT analyses of tibiae showed that the trabecular bone mass in Cthrc1-null mice was approximately $70 \%$ of that in wild-type mice (Figure $2 \mathrm{~A}$ and B). Bone histomorphometric and micro-CT analyses showed a significant decrease in trabecular number (Tb.N) and osteoblast number (Ob.N/BS) in Cthrc1-null mice, while trabecular thickness (Tb.Th) and tartrate-resistant acid phosphatase (TRAP)-positive osteoclast number (Oc.N/BS) had no change in Cthrc1-null mice (Figure 2C-E and Figure S4A). In addition, the osteoblast surface (Ob.S/BS) in Cthrc1-null mice, which represents the proportion of the bone surface covered with osteoblasts, was approximately $40 \%$ less than that in wild-type mice, and the osteoclast surface (Oc.S/ BS), which represents the proportion of the bone surface covered with osteoclasts, showed no significant difference between Cthrc1null and wild-type mice (Figure $2 \mathrm{~F}$ and Figure S4A). To analyze osteoblast proliferation in vivo, we performed BrdU incorporation assays in the calvaria of Cthrc1-null and wild-type littermates, and found that the percentage of proliferating cells was decreased by approximately 30\% in Cthrc1-null mice (Figure 2G). Moreover, double-labeling analyses with calcein, a marker of newly formed bone, showed that the bone formation rate was significantly decreased in Cthrc1-null mice (Figure 2H). Thus, these results indicate that the decreased bone mass in Cthrc1-null mice is due to the suppression of osteoblastic bone formation, not due to an acceleration of osteoclastic bone resorption.

\section{High bone mass and increased bone formation in transgenic mice overexpressing Cthrc1 in osteoblasts}

To determine if Cthrcl is sufficient to increase bone mass, we next generated transgenic mice that specifically overexpressed $3 \times$ HA tagged Cthrc1 in osteoblasts under the control of the Colla1 $2.3 \mathrm{~kb}$ osteoblast-specific promoter (Figure 3A). Immunohistochemistry with antibody against the HA-epitope tag showed specific expression of $3 \times \mathrm{HA}$ tagged protein in bone collars, periosteum, and trabecular bones (Figure 3B). Hemizygous Cthrc1 transgenic mice were born at the expected Mendelian ratio and grew normally in comparison with their wild-type littermates (data not shown). Skeletal preparations showed no apparent skeletal phenotypes in newborn hemizygous transgenic mice (Figure S2G). In addition, in situ hybridization analyses revealed that overexpression of Cthrcl in osteoblasts did not affect the expression of the osteoblast markers, Runx2 and Colla1, and chondrocyte markers, Col2a1 and Col10a1, in E16.5 mouse embryos (Figure $\mathrm{S} 3 \mathrm{~B})$. As we found an osteopenic phenotype in adult Cthrc1-null mice, we analyzed bone in adult Cthrc1 transgenic mice. Micro-CT analyses of tibiae showed that the trabecular bone mass in 

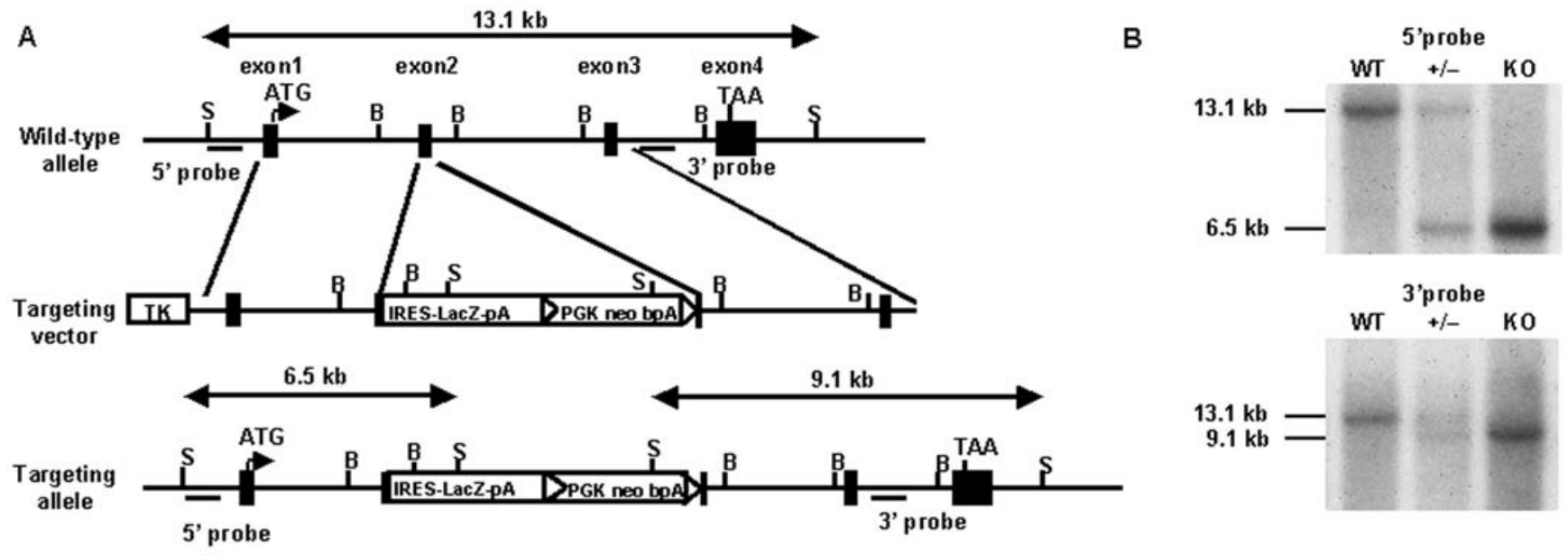

C

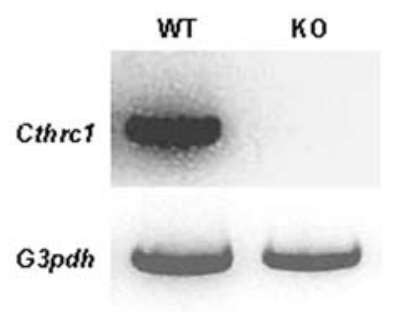

D

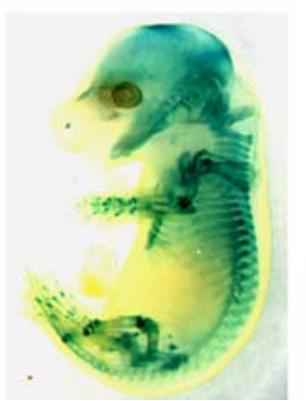

Figure 1. Generation of Cthrc1-null mice. (A) Structure of the genomic Cthrc1 locus, targeting vector, and targeting allele. Exons are depicted as closed boxes, and intronic sequences are shown as solid lines. IRES-LacZ-pA-loxP-flanked PGK-neo-bpA cassettes are depicted as open boxes. S, Sacl; B, BamHI. (B) Southern blot analysis of fetal genomic DNA. Genomic DNA isolated from the skin was digested with Sacl and then hybridized with the $5^{\prime}$ or $3^{\prime}$ probe. The wild-type and the mutant allele were detected with the $5^{\prime}$ probe as $13.1-\mathrm{kb}$ and $6.5-\mathrm{kb}$ fragments and with the $3^{\prime}$ probe as $13.1-\mathrm{kb}$ and 9.1-kb fragments, respectively. (C) RT-PCR analysis of Cthrc1 transcript in E16.5 wild-type and Cthrc1-null littermates. (D) Whole-mount X-gal staining of E16.5 heterozygous Cthrc1 embryo. WT: wild-type mice; KO: Cthrc1-null mice; +/-: Cthrc1 heterozygous mice.

doi:10.1371/journal.pone.0003174.g001

hemizygous Cthrc1 transgenic mice was approximately 25\% greater than that in wild-type mice (Figure $3 \mathrm{C}$ and D). Likewise, bone histomorphometric and micro-CT analyses showed significant increase in trabecular number (Tb.N) and osteoblast number (Ob.N/BS) in transgenic mice, while trabecular thickness (Tb.Th) and TRAP-positive osteoclast number (Oc.N/BS) had no change between transgenic mice and wild-type mice (Figure $3 \mathrm{E}-\mathrm{G}$ and Figure S4C). The osteoblast surface (Ob.S/BS) in transgenic mice was approximately $40 \%$ greater than that in wild-type mice, while there was no difference in the osteoclast surface (Oc.S/BS) between transgenic mice and wild-type mice (Figure $3 \mathrm{H}$ and Figure S4C). BrdU incorporation assays showed that the percentage of proliferating osteoblasts was increased by approximately $35 \%$ in transgenic mice (Figure 3I). Furthermore, doublelabeling analyses with calcein showed a marked increase in the bone formation rate in transgenic mice (Figure 3J). Thus, these data indicate that the increase in bone mass in Cthrc1 transgenic mice is due to the stimulation of osteoblastic bone formation.

\section{Cthrc1 stimulates osteoblast proliferation and differentiation}

Both the loss-of-function and gain-of-function analyses using mouse genetics approaches indicate that Cthrcl positively regulates osteoblastic bone formation in vivo. To clarify the functions of Cthrcl in osteoblasts, we isolated osteoblasts from the calvaria of wild-type (Cthrc1 $1^{+/+}$osteoblasts) and Cthrc1-null (Cthrc $1^{-1-}$ osteoblasts) newborn mice and analyzed the effects of Cthrcl on osteoblast proliferation and differentiation in vitro. BrdU incorporation in Cthrc $^{-/-}$osteoblasts was $40 \%$ less than that in Cthrc $^{+/+}$osteoblasts (Figure 4A). Real-time PCR analyses confirmed that the mRNA levels of the osteoblast marker genes, $A L P$, Col1a1, and Osteocalcin were all decreased in Cthrc $1^{-1-}$ osteoblasts, whereas the expression level of receptor activator of nuclear factor $\kappa \mathrm{B}$ ligand $(R A N K L)$, a major determinant of osteoclastogenesis, did not differ (Figure $4 \mathrm{~B}$ and Figure S4B). We further performed in vitro analyses of osteoprogenitor frequency and differentiation capacity by colony-forming unit (CFU) assays. GFU-ALP assays demonstrated that $C t h r c 1^{-1-}$ bone marrow cells had dramatically reduced osteoprogenitors, and mineralized area of CFU-osteoblast (CFU-O) was also reduced by more than half in $C t h r c 1^{-1-}$ bone marrow cells, consistent with decreased functional progenitors (Figure 4C and D). Likewise, we examined osteoblast proliferation and differentiation in osteoblasts derived from Cthrc1 transgenic mice (Tg-osteoblasts) in vitro. As shown in Figure 4A, BrdU incorporation in Tg-osteoblasts was $25 \%$ greater than that in wild-type osteoblasts. The mRNA levels 
A

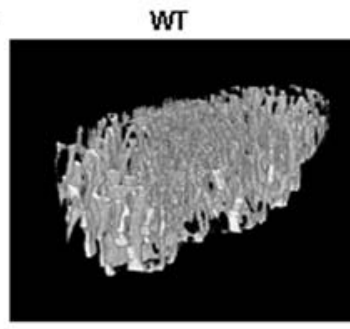

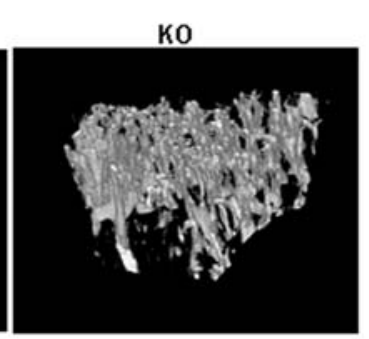

B

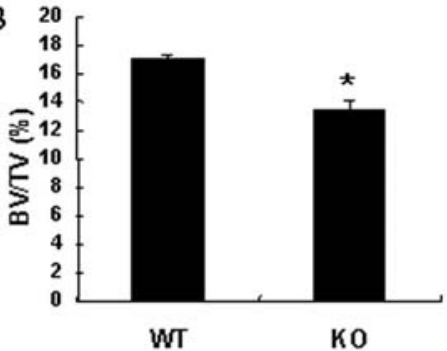

C

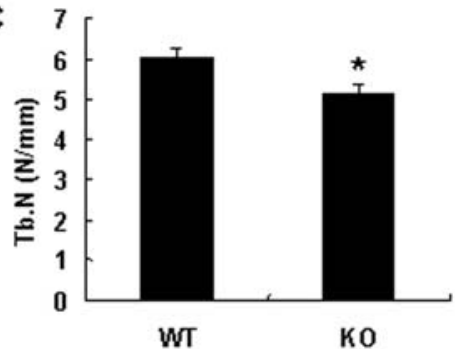

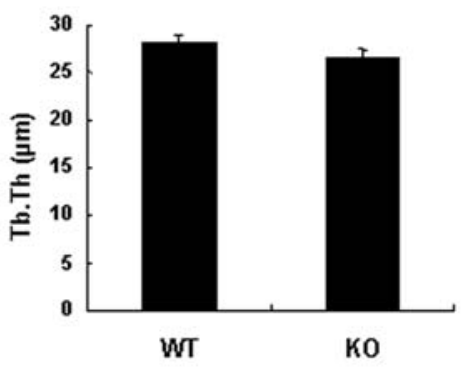

G

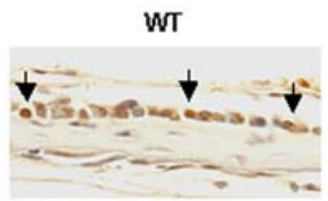

$14.9 \pm 1.7$

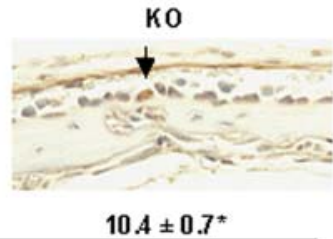

$10.4 \pm 0.7$
E

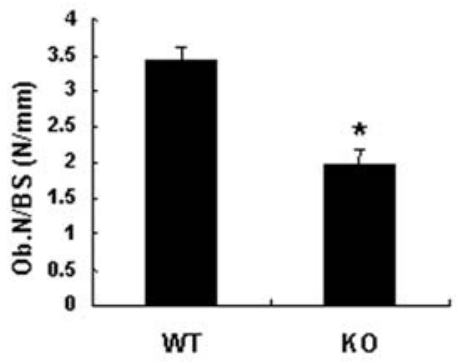

F

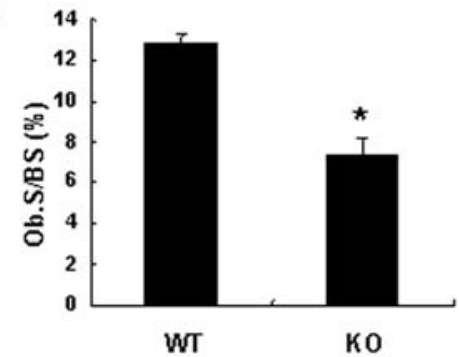

BrdU incorporation (\%)

$\mathrm{H}$

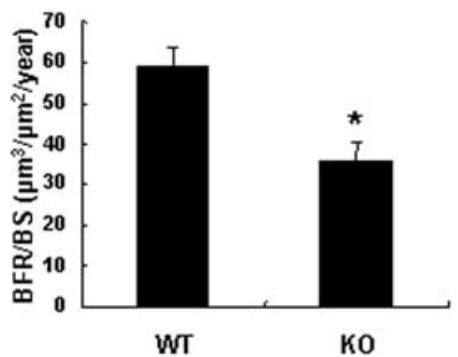

Figure 2. Bone phenotypes of Cthrc1-null mice. (A-D) Micro-CT analyses of tibiae in 2-month-old Cthrc1-null and wild-type mice ( $n=6)$. Bone volume/total volume (BV/TV), trabecular number (Tb.N), trabecular thickness (Tb.Th) are shown. (E and F) Bone histomorphometric analyses of vertebrae in 2-month-old Cthrc1-null and wild-type mice $(\mathrm{n}=6)$. Osteoblast number/bone surface (Ob.N/BS) and osteoblast surface/bone surface (Ob.S/BS) are shown. (G) Analyses of osteoblast proliferation in calvaria of 1-week-old Cthrc1-null and wild-type mice by BrdU incorporation assays. Arrows indicate BrdU positive osteoblasts. $(\mathrm{H})$ Calcein double-labeling of vertebrae in 2-month-old Cthrc1-null and wild-type mice. BFR : Bone formation rate. WT: wild-type mice; KO: Cthrc1-null mice. Data are shown as the mean \pm SEM $\left({ }^{*} p<0.05\right)$.

doi:10.1371/journal.pone.0003174.g002

of ALP, Col1a1, and Osteocalcin were all increased in Tg-osteoblasts, whereas the expression level of RANKL did not differ (Figure 4B and Figure S4D). CFU assays showed that the frequency of osteoprogenitors and mineralized area of CFU-O were significantly increased in Cthrc1 transgenic mice (Figure 4C and D). Thus, these results indicate that Cthrcl accelerates bone formation by stimulating osteoblast proliferation and differentiation, resulting in increased postnatal bone volume.

\section{Cthrc1 attenuates ovariectomy (OVX)-induced bone loss}

In view of an anabolic effect of Cthrcl on bone formation, we tested whether Cthrcl may impact bone mass in the OVX mice, an established preclinical disease model of postmenopausal osteoporosis [16]. Eight-week-old Cthrc1 transgenic and wild-type mice were ovariectomized or sham-operated, and four weeks after operation, metaphyseal regions of tibiae were analyzed by microCT. OVX induced $47 \%$ trabecular bone loss in wild-type mice, and $38 \%$, in transgenic mice (Figure $5 \mathrm{~A}$ and $\mathrm{B}$ ), and trabecular bone mass in OVX transgenic mice was only $17 \%$ less than that in sham-operated wild-type mice. Trabecular number, which was reduced by ovariectomy in both wild-type and transgenic mice, did not significantly differ between sham-operated wild-type mice and OVX transgenic mice (Figure 5C). Trabecular thickness, which was reduced in OVX wild-type mice, had no significant difference between sham-operated and OVX transgenic mice (Figure 5D). These results indicated that stimulatory effect of Cthrcl on bone formation partly prevents reduction in bone volume by ovariectomy. Indeed, increase in bone formation rates was maintained after ovariectomy in transgenic mice. (Figure 5E). Thus, Cthrcl attenuates estrogen deficiency-induced bone loss by enhanced osteoclastic bone resorption through stimulating osteoblastic bone formation.

\section{Discussion}

Bone mass is regulated by continuous remodeling which is based on the balanced action between osteoblastic bone formation and osteoclastic bone resorption [2]. This process is precisely coordinated to maintain the bone mass homeostasis mediated by multiple signaling pathways such as parathyroid hormone, transforming growth factor- $\beta$ (TGF- $\beta$ ) and BMP $[3,7]$. In this 
A

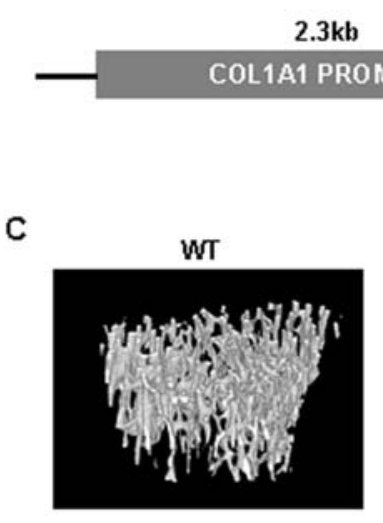

F

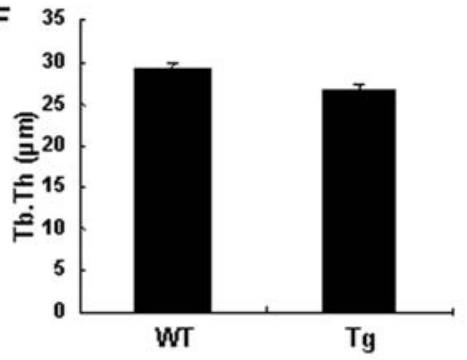

I

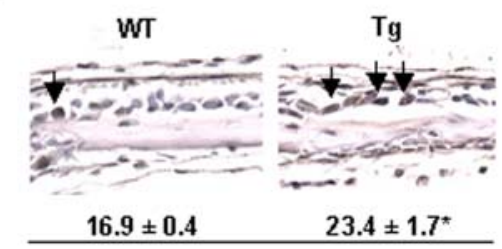

BrdU incorporation (\%)

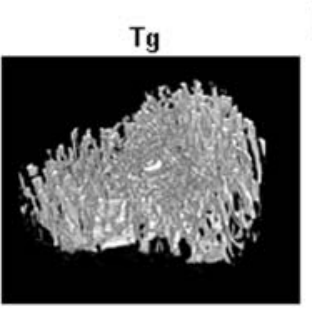

D

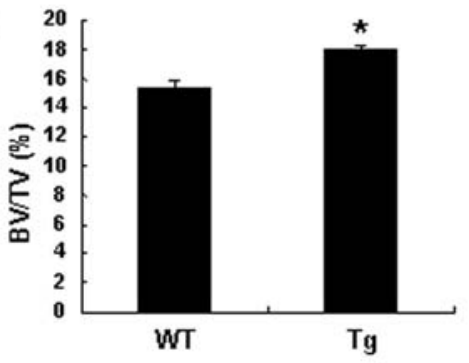

G

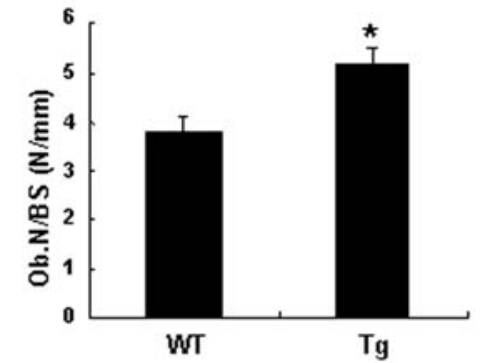

$\mathrm{J}$

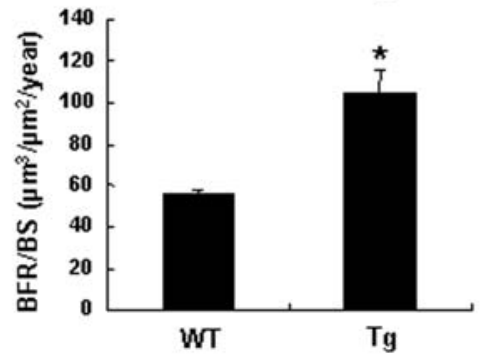

B

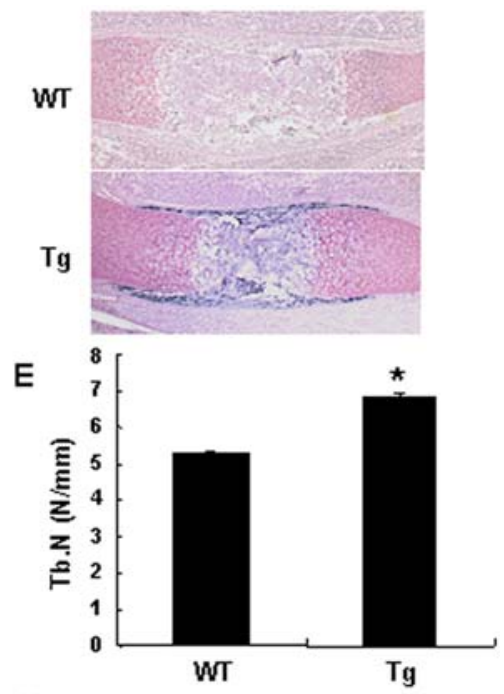

$\mathrm{H}$

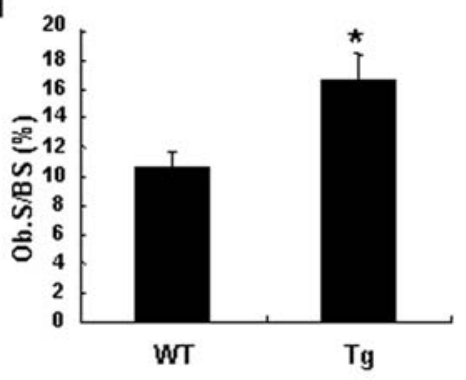

Figure 3. Generation of Cthrc1 transgenic mice and analyses of bone phenotypes. (A) Schematic representation of the Col1a1-Cthrc1 transgene. Poly A: SV40 polyadenylation signal. Arrows indicate the primers for genotyping. (B) Expression of $3 \times \mathrm{HA}$-tagged Cthrc1 protein in humeri of E16.5 Cthrc1 transgenic mice. (C-F) Micro-CT analyses of tibiae in 2-month-old Cthrc1 transgenic and wild-type mice $(n=6)$. Bone volume/total volume (BV/TV), trabecular number (Tb.N) and trabecular thickness (Tb.Th) are shown. (G and H) Bone histomorphometric analyses of vertebrae in 2month-old Cthrc1 transgenic and wild-type mice $(n=6)$. Osteoblast number/bone surface (Ob.N/BS) and osteoblast surface/bone surface $(\mathrm{Ob} . \mathrm{S} / \mathrm{BS})$ are shown. (I) Analyses of osteoblast proliferation in calvaria of 1-week-old Cthrc1 transgenic and wild-type mice by BrdU incorporation assays. Arrows indicate BrdU positive osteoblasts. (J) Calcein double-labeling of vertebrae in 2-month-old Cthrc1 transgenic and wild-type mice. BFR: Bone formation rate. WT: wild-type mice; Tg: Cthrc1 transgenic mice. Data are shown as the mean \pm SEM ( $\left.{ }^{*} p<0.05\right)$.

doi:10.1371/journal.pone.0003174.g003

study, we show that Cthrcl is involved in postnatal bone formation. In Cthrc1-null mice, bone volume, trabecular number, osteoblast number, and bone formation rates were significantly decreased. In Cthrc1 transgenic mice, bone volume, trabecular number, osteoblast number and bone formation rates were increased. In vitro, CFU assays in bone marrow cells and analyses of expression of osteoblast specific genes, ALP, Colla1, and Osteocalcin, in osteoblasts revealed that Cthrcl stimulates differentiation of osteoprogenitor cells and osteoblasts. Furthermore, BrdU incorporation assays showed that Cthrcl stimulates osteoblast proliferation in vitro and in vivo. In contrast, Cthrcl did not affect either TRAP-positive osteoclast number and osteoclast surface in vivo, or RANKL expression levels in primary osteoblasts. These findings indicate that Cthrcl acts as a stimulator of osteoblastic bone formation, but has no effect on osteoclastic bone resorption.
BMPs have potent osteogenic effects and control osteoblast proliferation and differentiation during osteogenesis [6,12,17]. Several studies of cell-specific inactivation or activation of BMP signals in mice have shown that BMPs regulate postnatal bone remodeling [8-12]. As shown in previous studies, Cthrcl is regulated by TGF- $\beta$ and BMP4, and a putative Smad binding site is identified in the promoter region of Cthrc1 [13,18]. We show that Cthrc1, which is induced by BMP2, is not required for skeletal development, but is for the maintainance of bone homeostasis, suggesting that Cthrcl may act as one of the downstream targets of BMP-Smad signaling, followed by enhanced expression of osteoblast specific genes including ALP, Col1a1 and Osteocalcin.

The detail mechanism of Cthrcl during bone formation is unclear. Cthrcl inhibited TGF- $\beta$ signaling in smooth muscle cells but not in endotherial cells [14]. Contrary to the effect of Cthrcl on osteoblasts in Colla1 expression in our study, overexpression of 
A
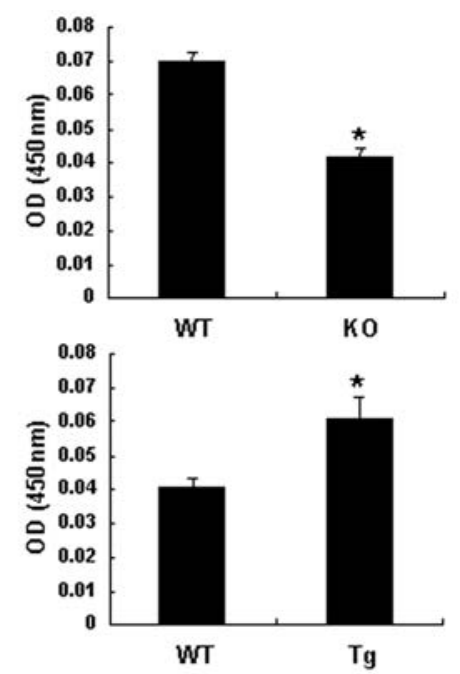

B
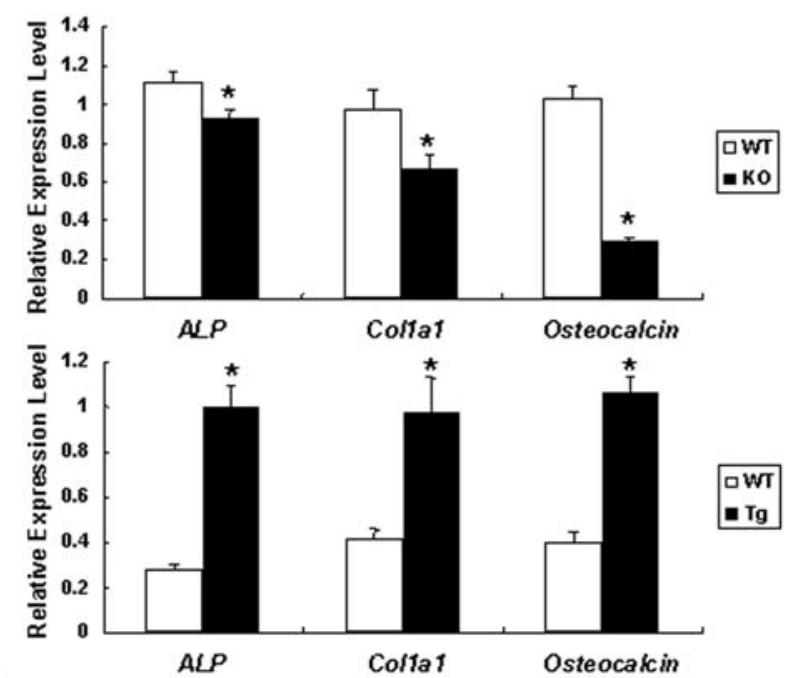

C
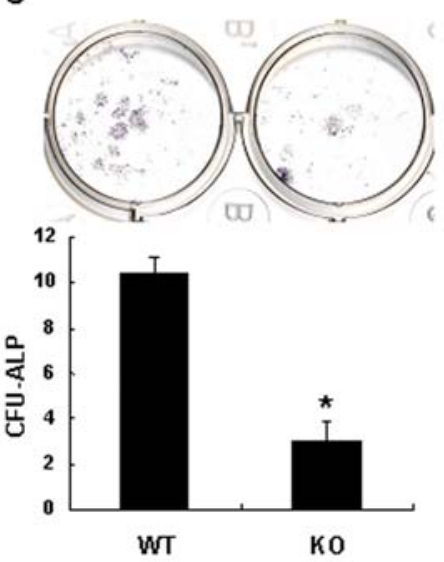
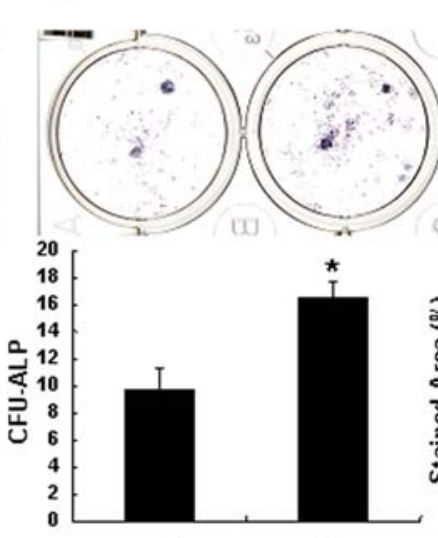

WT

$\operatorname{Tg}$
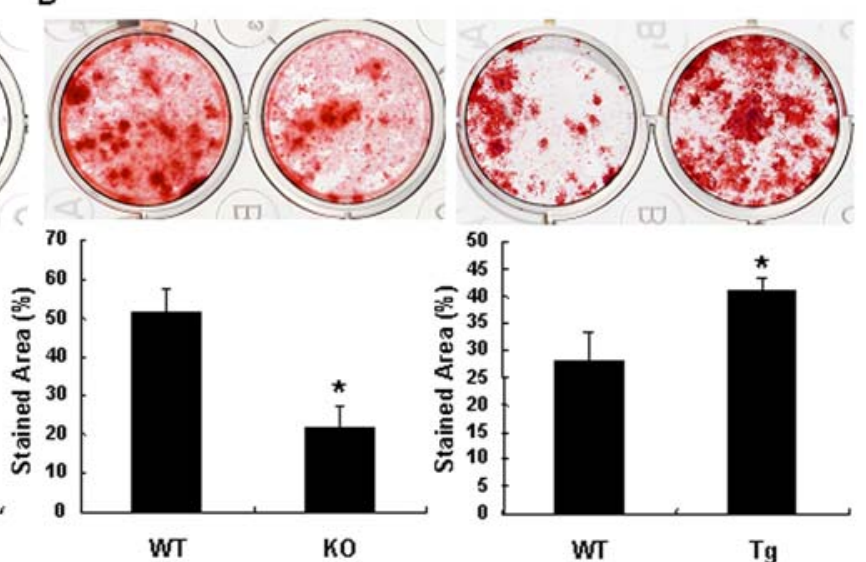

Figure 4. Effects of Cthrc1 on osteogenesis. (A) Cell proliferation in primary osteoblasts harvested from Cthrc 1-null mice (upper panel) and from Cthrc1 transgenic mice (lower panel) as shown by BrdU incorporation assays. (B) Expression of early and late osteoblast marker genes in primary osteoblasts harvested from Cthrc1-null mice (upper panel) and Cthrc1 transgenic mice (lower panel). (C) The total numbers of CFU-ALP in bone marrow cell cultures derived from Cthrc1-null mice (left) and Cthrc1 transgenic mice (right). (D) The mineralized area of CFU-O in bone marrow cell cultures derived from Cthrc1-null mice (left) and Cthrc1 transgenic mice (right). WT: wild-type mice; KO: Cthrc1-null mice; Tg: Cthrc1 transgenic mice. Data are shown as the mean \pm SEM ( $\left.{ }^{*} p<0.05\right)$.

doi:10.1371/journal.pone.0003174.g004

Cthrcl in PACl cells, smooth muscle cell line, reduced mRNA levels of Col1a1, and collagen deposition [13,14]. These results suggest that the function of Cthrcl differs among cell types, or that Cthrcl signals through its own signaling pathway which is functional in a cell-type specific manner. In addition, there are discrepancies of phenotypes between $C t h r c 1$ transgenic mice which we generated in this study and the transgenic mice overexpressing Cthrc1 previously reported [14]. First, transgenic mice we generated display a high-bone-mass phenotype, whereas transgenic mice previously reported show an osteopenic phenotype. Second, transgenic mice previously reported are smaller in size and have a higher rate of postnatal mortality, whereas transgenic mice we generated are fertile and normal in appearance and body weight. A possible explanation for these discrepancies is that transgenic mice we generated overexpress Cthrc1 specifically in osteoblasts under the control of the Colla1 $2.3 \mathrm{~kb}$ osteoblast-specific promoter to focus on the function of Cthrcl in bones, while transgenic mice previously reported ubiquitously overexpress Cthrc1 under the control of cytomegalovirus promoter.

Ovariectomy leads to a deficit in bone mineral densities due to cortical bone modeling and resorption of cancellous bone [19,20]. This change largely results in severe cancellous osteopenia.
Dempster et al. demonstrated that the primary mechanism of ovariectomy-induced bone loss is osteoclast perforation and removal of the trabecular plates [21]. Furthermore, a recent study showed that selective ablation of estrogen receptor $\alpha$ in differentiated osteoclasts induced trabecular bone loss, similar to the osteoporotic bone phenotype, caused by inhibition of osteoclast apoptosis [22]. Thus, ovariectomy-induced bone loss, a model of postmenopausal osteoporosis, results from an acceleration of osteoclastic bone resorption, followed by high bone turnover in which bone resorption exceeds bone formation. Cthrc1-null mice exhibit cancellous bone loss, and Cthrc1 transgenic mice increase cancellous bone with no change in osteoclastic bone resorption. In addition, Cthrcl stimulates osteoblast proliferation and differentiation and increases bone formation rates, suggesting that Cthrcl prevents cancellous bone loss in ovariectomized mice by stimulating osteoblastic bone formation. Indeed, bone volume and trabecular number in OVX transgenic mice were higher than those in OVX wild-type mice, and the loss of trabecular thickness by ovariectomy was protected in transgenic mice. Moreover, increased bone formation rates in transgenic mice were maintained after OVX. Considering that Cthrcl does not affect 
A
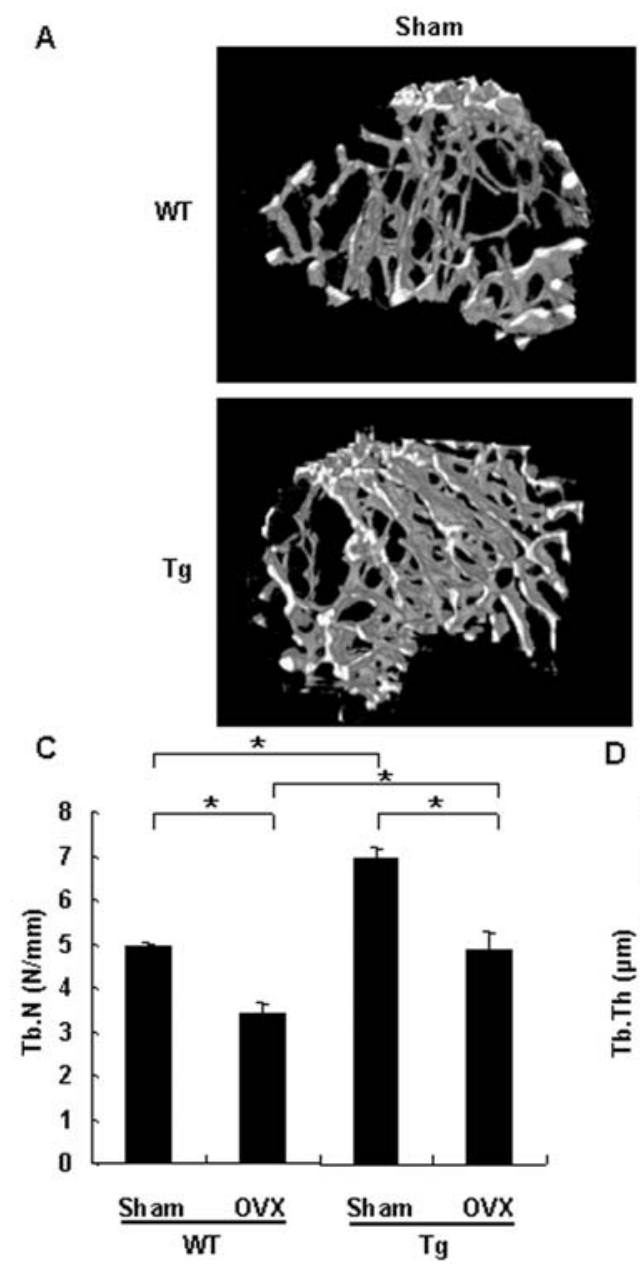
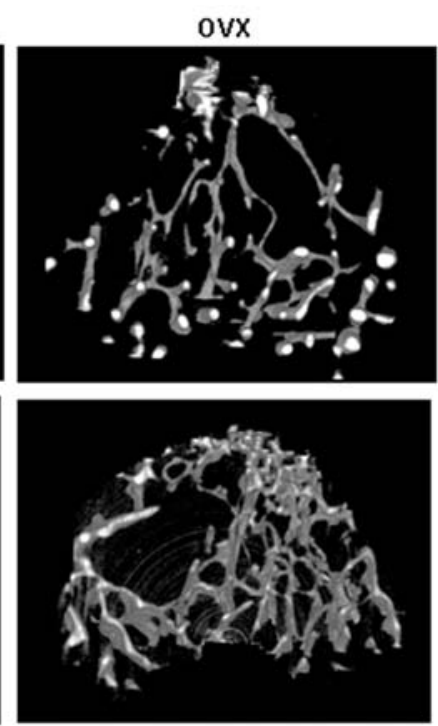

D

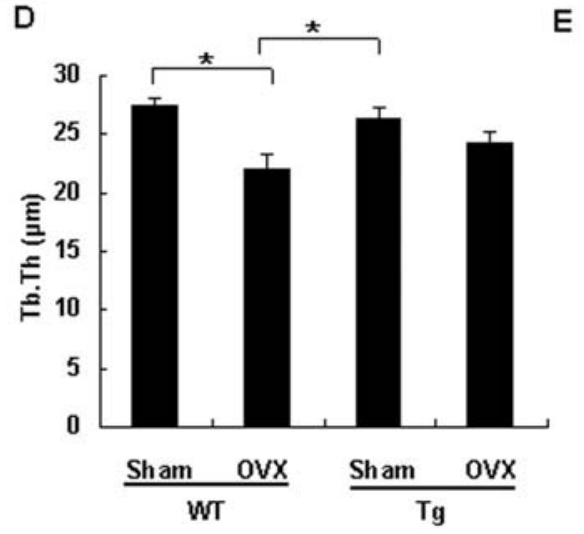

B

E
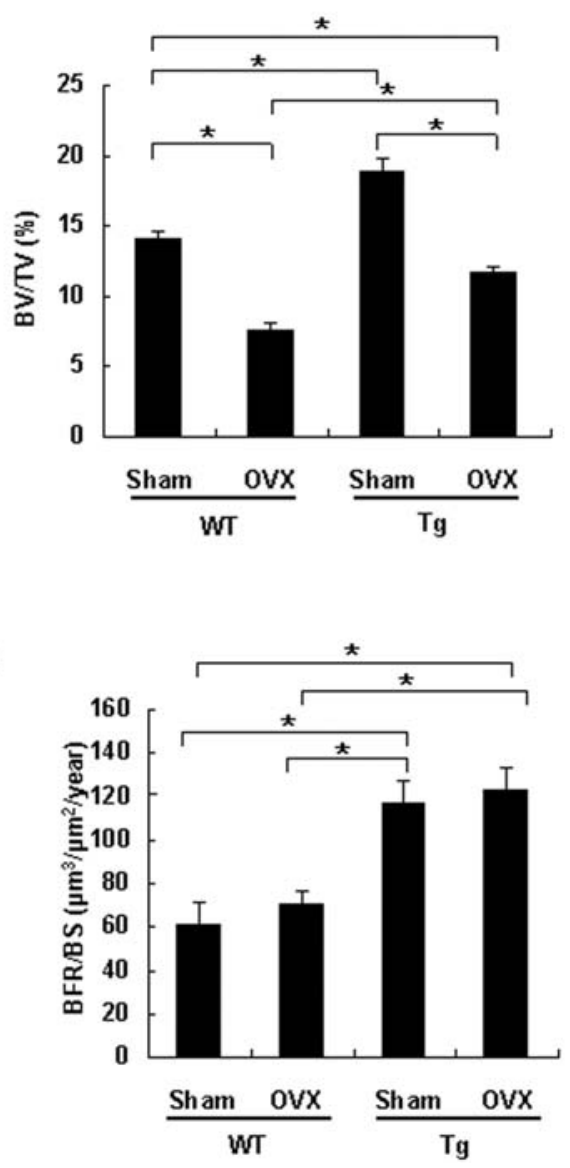

Figure 5. OVX-induced bone loss is attenuated in Cthrc1 transgenic mice. (A) micro-CT of tibiae from 3-month-old Cthrc1 transgenic and wild-type mice which were sham-operated or ovariectomized at 2 months of age $(n=5)$. (B-E) Bone volume/total volume (BV/TV) (B), trabecular number (Tb.N) (C), trabecular thickness (Tb.Th) (D) and bone formation rate (BFR) (E) were assessed by micro-CT or bone histomorphometry. WT: wildtype mice; Tg: Cthrc1 transgenic mice. Data are shown as the mean \pm SEM ( $\left.{ }^{*} p<0.05\right)$.

doi:10.1371/journal.pone.0003174.g005

osteoclastogenesis, enhanced bone formation by overexpression of Cthrcl may attenuate bone loss caused by enhanced osteoclastic bone resorption induced by OVX.

It is noteworthy in this article that Cthrcl may have a potential role in treating osteoporosis, because we observed that osteoblastspecific overexpression of Cthrc1 in mice attenuates bone loss which is induced by OVX, an accepted preclinical disease model of postmenopausal osteoporosis. The function of Cthrcl as a stimulator of bone formation without affecting bone resorption is very promising, because most types of drugs which are currently used for the treatment of osteoporosis are antiresorptive in nature and insufficient for restoring bone volume in osteoporotic patients [23]. Although anabolic agents improve bone mass by stimulating osteoblast-mediated bone formation, only a single anabolic agent, parathyroid hormone $1-34$ is available to treat osteoporosis at present [24,25]. Herein, we indicate that Cthrcl attenuates estrogen deficiency-induced bone loss by stimulating osteoblastic bone formation. Hence, Cthrcl constitutes a potential target of an anabolic therapy for osteoporosis and the therapeutic effects are expected to be enhanced with antiresorptive drugs.

\section{Materials and Methods}

Suppression subtractive hybridization

ATDC5 cells were cultured for a total of 5 days in DMEM/ Ham's F12 medium supplemented with 5\% FBS and $10 \mu \mathrm{g} / \mathrm{ml}$ insulin with medium change every other day. Cells were exposed to $1 \mu \mathrm{g} / \mathrm{ml} \mathrm{BMP2}$ or vehicle for $10 \mathrm{hr}$. Poly $(\mathrm{A})^{+} \mathrm{RNA}$ was isolated from BMP2 -untreated and BMP2 -treated ATDC5 cells by a single-step method as previously described [26] and analyzed by suppression subtractive hybridization according to the manufacturer's instruction (PCR-Select cDNA Subtractions Kit, Glontech). After subtraction, the cDNAs were ligated into pCR2.1 (Invitrogen), and these subtracted cDNA libraries were further screened by differential hybridization (differential screening kit, Clontech). The cDNA fragment of approximately 500-bp expressed at a high level in BMP2 -treated ATDC5 cells was identified. Oligo (dT) primed cDNA library from poly $(\mathrm{A})^{+} \mathrm{RNA}$ of $\mathrm{BMP} 2$-treated ATDC5 cells was constructed in $\lambda$ ZAP Express vector (Stratagene), and $1 \times 10^{6}$ plaques were screened with the 500-bp fragment as a probe as previously described [26]. 


\section{Generation of mutant mice}

A Cthrcl genomic clone was isolated from a mouse 129SvEv genomic DNA library. The targeting vector was constructed by inserting an IRES-LacZ-pA-loxP-flanked neomycin resistance expression cassette into exon 2 of the Cthrc1 gene. Homologous recombination in $129 \mathrm{SvEv}$ ES clones harboring Protamine 1-Cre transgenes was identified by Southern blot analysis of SacIdigested genomic DNA using $5^{\prime}$ and $3^{\prime}$ probes located outside the homology regions used for gene recombination [27]. Mouse chimeras were generated by C57BL/ 6 host blastocyst injection of mutant embryonic stem cell clones, and the chimeras obtained were bred with C57BL/6 mice to generate heterozygous Cthrc1 mice. Heterozygous mutants were then backcrossed eight times with C57BL/6 mice to generate mutant mice with a C57BL/6 genetic background. We then performed RT-PCR to determine the presence of Cthrc1 transcripts. We used the following primers of Cthrc1 for RT-PCR, based on exon3 and exon4: 5'-CTGCGAGTTCTGTTCAGTGG-3' and 5'-GGGACTGAAATCGTCAGAGG-3'. Cthrc1 transgenic mice were generated using an osteoblast-specific 2.3-kb Col1a1 promoter, the $1.25-\mathrm{kb} 3 \times \mathrm{HA}$ tagged full-length mouse Cthrc1 cDNA, and the 240-bp SV40 polyadenylation signal [28]. DNAs were injected into pronuclei of fertilized C57BL/ $6 \times \mathrm{DBA} / 2$ hybrid eggs, and the injected eggs were then transferred into $\mathrm{CD} 1$ foster mothers. Hemizygous mutants were backcrossed eight times with $\mathrm{C} 57 \mathrm{BL} / 6$ mice to generate transgenic mice with a C57BL/6 genetic background. The tissue specificity of transgene expression was examined in immunohistochemistry studies. Routine mouse genotyping was performed by PCR. The following primer pairs were used: $5^{\prime}$ CATCAAGATGGTATAAAAGG-3' and 5'-GCAGCAGCAGCACAAGGAAG-3'. The experimental protocols were approved by the Animal Care and Use Committee of Kyoto University.

\section{Histological analyses}

Whole mount X-gal staining of embryos was performed as previously described [29]. For the histological analyses, we fixed embryos with $4 \%$ paraformaldehyde, embedded them in paraffin, and sectioned them into $7-\mu \mathrm{m}$-thick slices. Immunohistochemical staining was performed using peroxidase chromogens (Zymed)/ TrueBlue substrate (KPL) with rabbit polyclonal anti-HA antibody (1:500, Covance). For in vivo BrdU labeling, 1-week-old mice were injected intraperitoneally with $100 \mu \mathrm{g} / \mathrm{kg} \operatorname{BrdU}$ (Amersham) and sacrificed $3 \mathrm{hr}$ later. Sections were stained with anti-BrdU antibody (Amersham) and were counterstained with hematoxylin according to the manufacturer's instruction.

\section{Micro-CT Analyses}

High-resolution micro-CT scanning (SMX-100CT; Shimazu) was performed to measure morphological indices of metaphyseal regions of tibia as previously described [30]. Metaphyseal regions were scanned 100 times with a slice increment of $8 \mu \mathrm{m}$. The most proximal slice was defined as the plane where the growth plate had just disappeared. Material properties were calculated using a commercial software package (VG Studio Max1.2: Visual Science) [30].

\section{Bone Histomorphometry}

The mice were injected subcutaneously with calcein $(20 \mathrm{mg} / \mathrm{kg}$ body weight; Sigma) 10 and 3 days before sacrifice. Fifth lumber vertebrae were fixed with $4 \%$ paraformaldehyde for $18 \mathrm{hr}$ at $4^{\circ} \mathrm{C}$. Undecalcified bones were embedded in methylmethacrylate, and $4-\mu \mathrm{m}$-thick sections were prepared for bone histomorphometric analyses of adult mice as previously described [31]. Sections were stained with $1 \%$ toluidine blue. Static and dynamic histomorphometric analyses were performed according to standard protocols using Histometry RT (SYSTEM-SUPPLY) [32,33].

\section{Osteoblast Isolation and Culture}

Primary osteoblasts were harvested from calvaria of newborn mice by sequential collagenase digestion (Roche) and were maintained in $\alpha$-MEM containing $10 \%$ FBS. BrdU incorporation was measured using the cell proliferation ELISA Biotrack kit (Amersham).

\section{Real-Time PCR}

Primary osteoblasts were cultured in $\alpha$-MEM containing $10 \%$ FBS, $50 \mu \mathrm{g} / \mathrm{ml}$ ascorbic acid, and $10 \mathrm{nM} \beta$-glycerophosphate for 10 days, and total RNA was isolated from the cultured cells by RNeasy Mini Kit (Qiagen) according to the manufacturer's instruction. Two $\mu \mathrm{g}$ of total RNA was reverse transcribed to cDNA with the use of Transcriptor First Strand cDNA Synthesis Kit (Roche). Real-time PCR was performed using the LightCycler system with the FastStart DNA Master SYBR Green (Roche). The following primers were used: $G 3 p d h, 5^{\prime}$-TGTCGGTCGTGGATCTGAC-3' and 5'-CGTGGTTCACGACGTTCTTG-3'; ALP, 5'-ACTCAGGGCAATGAGGTCAC- ${ }^{\prime}$ and 5'-CACCCGAGTGGTAGTCACAA -3'; Col1a1, 5'-CTCCTGGCAAGAATGGAGAT-3' and 5'-AATCGACGAGCACCGTGA-3'; Osteocalcin, 5' AGACTCGGGCGCTACCTT-3' and 5'-CTCGTCACAAGCAGGGTTAAG-3'.

\section{Measurement of CFU-ALP and CFU-O}

Bone marrow cells were isolated as previously described [26]. Bone marrow cells from 5 -week-old mice were plated into 12 -well plates at $2.5 \times 10^{6}$ cells per well for CFU-ALP assays and at $5 \times 10^{6}$ cells per well for CFU-O assays, and were cultured in $\alpha$ MEM containing $10 \% \mathrm{FBS}, 50 \mu \mathrm{g} / \mathrm{ml}$ ascorbic acid, and $10 \mathrm{nM}$ $\beta$-glycerophosphate. For CFU-ALP assays, cultures were stained at day 10 with Sigma alkaline phosphatase kit (Sigma), and colonies with $>20$ cells were counted. Cultures were stained with $1 \%$ alizarin red $\mathrm{S}$ (Wako) at day 20 for the CFU-O assay of Cthrc1null mice, and at day 16 for that of Cthrc1 transgenic mice, respectively. The stained area was calculated using the freeware Image-J (NIH).

\section{Ovariectomy}

Mice were ovariectomized or sham-operated at 2 months of age, and all of the mice were killed 4 weeks later and the tibiae and vertebrae were removed for analyses.

\section{Statistical analyses}

Statistical analysis was performed by Student's t test to determine the significance between groups. Wild-type and transgenic OVX and sham-operated mice were analyzed by ANOVA followed by Fisher's protected least significant difference. Values were considered statistically significant at $p<0.05$.

More methods were shown in Supporting Information Text S1.

\section{Supporting Information}

Figure S1 Analyses of Cthrcl expression in vitro and in vivo by northern blot and in situ hybridization. (A) Effect of BMP2 (1 $\mu \mathrm{g}$ / $\mathrm{ml}$ ) on Cthrcl expression in ATDC5 cells. Cthrcl expression is upregulated by BMP2. (B) Expression of Cthrcl in various cell lines. (C) Expression of Cthrcl in adult mouse tissues. (D) In situ hybridization analysis of Cthrcl expression in limb buds of E13.5 
and E16.5 mouse embryos. (E) Expression of Cthrcl during embryogenesis.

Found at: doi:10.1371/journal.pone.0003174.s001 (8.89 MB TIF)

Figure S2 Skeletal preparation of Cthrc1-null and Cthrcl transgenic mice. (A) Whole-mount X-gal staining of heterozygous Cthrcl embryos during embryogenesis. (B and C) Skeletons of newborn Cthrcl-null mice $(\mathrm{B})$ and Cthrcl transgenic mice $(\mathrm{C})$ stained by alcian blue followed by alizarin red. WT: wild-type mice; KO: Cthrcl-null mice; Tg: Cthrcl transgenic mice. Found at: doi:10.1371/journal.pone.0003174.s002 (5.91 MB TIF)

Figure S3 In situ hybridization analyses of osteoblast and chondrocyte marker genes in Cthrcl-null and Cthrcl transgenic mouse embryos. Runx2, Colla1, Col2al and Coll0al expression in humeri of E16.5 embryos. (A) Cthrcl-null mouse embryos. (B) Cthrcl transgenic mouse embryos. WT: wild-type mice; KO: Cthrc1-null mice; Tg: Cthrcl transgenic mice.

Found at: doi:10.1371/journal.pone.0003174.s003 (10.15 MB TIF)

Figure S4 Effect of Cthrcl on osteoclastogenesis. (A) TRAP staining of vertebrae of 2-month-old Cthrcl-null and wild-type mice. TRAP-positive osteoclast number/bone surface (Oc.N/BS) and osteoclast surface/bone surface (Oc.S/BS) are shown $(\mathrm{n}=6)$. (B) Expression of RANKL in primary osteoblasts harvested from

\section{References}

1. Olsen BR, Reginato AM, Wang W (2000) Bone development. Annu Rev Cell Dev Biol 16: 191-220.

2. Karsenty G, Wagner EF (2002) Reaching a genetic and molecular understanding of skeletal development. Dev Cell 2: 389-406.

3. Harada S, Rodan GA (2003) Control of osteoblast function and regulation of bone mass. Nature 423: 349-355.

4. Hughes FJ, Collyer J, Stanfield M, Goodman SA (1995) The effects of bone morphogenetic protein-2, -4 , and -6 on differentiation of rat osteoblast cells in vitro. Endocrinology 136: 2671-2677.

5. Abe E, Yamamoto M, Taguchi Y, Lecka-Czernik B, O'Brien CA, et al. (2000) Essential requirement of BMPs-2/4 for both osteoblast and osteoclast formation in murine bone marrow cultures from adult mice: antagonism by noggin. J Bone Miner Res 15: 663-673.

6. Kronenberg HM (2003) Developmental regulation of the growth plate. Nature 423: $332-336$.

7. Abe E (2006) Function of BMPs and BMP antagonists in adult bone. Ann N Y Acad Sci 1068: 41-53.

8. Devlin RD, Du Z, Pereira RC, Kimble RB, Economides AN, et al. (2003) Skeletal overexpression of noggin results in osteopenia and reduced bone formation. Endocrinology 144: 1972-1978.

9. Wu XB, Li Y, Schneider A, Yu W, Rajendren G, et al. (2003) Impaired osteoblastic differentiation, reduced bone formation, and severe osteoporosis in noggin-overexpressing mice. J Clin Invest 112: 924-934

10. Zhao M, Harris SE, Horn D, Geng Z, Nishimura R, et al. (2002) Bone morphogenetic protein receptor signaling is necessary for normal murine postnatal bone formation. pp 1049-1060.

11. Mishina Y, Starbuck MW, Gentile MA, Fukuda T, Kasparcova V, et al. (2004) Bone morphogenetic protein type IA receptor signaling regulates postnatal osteoblast function and bone remodeling. J Biol Chem 279: 27560-27566.

12. Yoshida Y, Tanaka S, Umemori H, Minowa O, Usui M, et al. (2000) Negative regulation of BMP/Smad signaling by Tob in osteoblasts. Cell 103: 1085-1097.

13. Pyagay P, Heroult M, Wang Q, Lehnert W, Belden J, et al. (2005) Collagen triple helix repeat containing 1 , a novel secreted protein in injured and diseased arteries, inhibits collagen expression and promotes cell migration. Circ Res 96: 261-268.

14. LeClair RJ, Durmus T, Wang Q, Pyagay P, Terzic A, et al. (2007) Cthrcl is a novel inhibitor of transforming growth factor-beta signaling and neointimal lesion formation. Circ Res 100: 826-833.

15. Durmus T, LeClair RJ, Park KS, Terzic A, Yoon JK, et al. (2006) Expression analysis of the novel gene collagen triple helix repeat containing-1 (Cthrcl). Gene Expr Patterns 6: 935-940.

16. Kalu DN (1991) The ovariectomized rat model of postmenopausal bone loss. Bone Miner 15: 175-191.

17. Wozney JM, Rosen V, Celeste AJ, Mitsock LM, Whitters MJ, et al. (1988) Novel regulators of bone formation: molecular clones and activities. Science 242 . 1528-1534.
Cthrcl-null mice, assessed by real-time PCR. (C) TRAP staining of vertebrae of 2-month-old Cthrcl transgenic and wild-type mice. TRAP-positive osteoclast number/bone surface (Oc.N/BS) and osteoclast surface/bone surface (Oc.S/BS) are shown $(n=6)$. (D) Expression of RANKL in primary osteoblasts harvested from Cthrcl transgenic mice, assessed by real-time PCR. WT: wild-type mice; KO: Cthrcl-null mice; Tg: Cthrcl transgenic mice. Data are shown as the mean \pm SEM $(* \mathrm{p}<0.05)$.

Found at: doi:10.1371/journal.pone.0003174.s004 (9.83 MB TIF)

Text S1 Supplementary Methods.

Found at: doi:10.1371/journal.pone.0003174.s005 (0.05 MB DOG)

\section{Acknowledgments}

We thank Janie Finch for editorial assistance, Neung-Seon Seo and Magnus Hook for technical support, and Steve O'Gorman for PC3 mouse ES cells.

\section{Author Contributions}

Conceived and designed the experiments: BdG HA. Performed the experiments: HK KMK BGD HA. Analyzed the data: HK BGD RRB TN HA. Contributed reagents/materials/analysis tools: ZZ JMD RRB BdC. Wrote the paper: HK BdC HA.

18. Tang L, Dai DL, Su M, Martinka M, Li G, et al. (2006) Aberrant expression of collagen triple helix repeat containing 1 in human solid cancers. Clin Cancer Res 12: 3716-3722.

19. Wronski TJ, Cintron M, Dann LM (1988) Temporal relationship between bone loss and increased bone turnover in ovariectomized rats. Calcif Tissue Int 43: 179-183.

20. Turner RT, Vandersteenhoven JJ, Bell NH (1987) The effects of ovariectomy and 17 beta-estradiol on cortical bone histomorphometry in growing rats. J Bone Miner Res 2: 115-122.

21. Dempster DW, Birchman R, Xu R, Lindsay R, Shen V (1995) Temporal changes in cancellous bone structure of rats immediately after ovariectomy. Bone 16: 157-161.

22. Nakamura T, Imai Y, Matsumoto T, Sato S, Takeuchi K, et al. (2007) Estrogen prevents bone loss via estrogen receptor alpha and induction of Fas ligand in osteoclasts. Cell 130: 811-823.

23. Rodan GA, Martin TJ (2000) Therapeutic approaches to bone diseases. Science 289: 1508-1514.

24. Canalis E, Giustina A, Bilezikian JP (2007) Mechanisms of anabolic therapies for osteoporosis. N Engl J Med 357: 905-916.

25. Garrett IR (2007) Anabolic agents and the bone morphogenetic protein pathway. Curr Top Dev Biol 78: 127-171.

26. Kimura H, Akiyama H, Nakamura T, de Crombrugghe B (2007) Tenascin-W inhibits proliferation and differentiation of preosteoblasts during endochondral bone formation. Biochem Biophys Res Commun 356: 935-941.

27. O'Gorman S, Dagenais NA, Qian M, Marchuk Y (1997) Protamine-Cre recombinase transgenes efficiently recombine target sequences in the male germ line of mice, but not in embryonic stem cells. Proc Natl Acad Sci U S A 94: 14602-14607.

28. Rossert J, Eberspaecher H, de Crombrugghe B (1995) Separate cis-acting DNA elements of the mouse pro-alpha 1(I) collagen promoter direct expression of reporter genes to different type I collagen-producing cells in transgenic mice. J Cell Biol 129: 1421-1432.

29. Nakashima K, Zhou X, Kunkel G, Zhang Z, Deng JM, et al. (2002) The novel zinc finger-containing transcription factor osterix is required for osteoblast differentiation and bone formation. Cell 108: 17-29.

30. Nakanishi R, Shimizu M, Mori M, Akiyama H, Okudaira S, et al. (2006) Secreted frizzled-related protein 4 is a negative regulator of peak BMD in SAMP6 mice. J Bone Miner Res 21: 1713-1721.

31. Hahn M, Vogel M, Delling G (1991) Undecalcified preparation of bone tissue: report of technical experience and development of new methods. Virchows Arch A Pathol Anat Histopathol 418: 1-7.

32. Parfitt AM (2002) Physiologic and pathogenetic significance of bone histomorphometric data. In: Coe FL, Favus MJ, eds. Disorders of Bone Miner Metabolism, 2nd ed. Philadelphia, PA, USA: Lippincott Williams \& Wilkins. pp 469-485.

33. Parfitt AM, Drezner MK, Glorieux FH, Kanis JA, Malluche H, et al. (1987) Bone histomorphometry: standardization of nomenclature, symbols, and units. Report of the ASBMR Histomorphometry Nomenclature Committee. J Bone Miner Res 2: 595-610. 